\title{
Characteristics of the Japanese Diet Described in Epidemiologic Publications: A Qualitative Systematic Review
}

\author{
Nozomu SuZukI ${ }^{1, *}$, Yoshihito Goto ${ }^{1}$, Haruka OTA ${ }^{1}$, Kumiko KITO ${ }^{1}$, Fumika MANO ${ }^{2}$, \\ Erina $\mathrm{JOO}^{2}$, Kaori IKEDA ${ }^{2}$, Nobuya INAGAKI ${ }^{2}$ and Takeo NAKAYAMA ${ }^{1}$ \\ ${ }^{1}$ Department of Health Informatics, School of Public Health, Kyoto University, Kyoto 606-8501, Japan \\ ${ }^{2}$ Department of Diabetes, Endocrinology and Nutrition, Graduate School of Medicine, \\ Kyoto University, Kyoto 606-8507, Japan
}

(Received June 30, 2017)

\begin{abstract}
Summary Context: International interest in the Japanese diet has grown in recent years. Objective: The aim of this systematic review was to evaluate and organize the Japanese diet and dietary characteristics from an epidemiological perspective, mainly focusing on the nutritional and dietary elements. Data Sources: PubMed, Web of Science, Japan Medical Abstracts Society, JDream III, and CiNii databases were searched. Study Selection: The eligibility criteria included research with an epidemiological study design that was either crosssectional, cohort, or case-control-based that defined the dietary patterns of the Japanese diet using dietary pattern analysis. A total of 39 research articles that described the Japanese diet were included. Data Extraction: The data that were extracted included the following: implementing country, location, study design, participant characteristics, key outcomes, methods used in the analysis of dietary patterns, and descriptions of the Japanese diet. Data Synthesis: As a result of the systematic review analyzing the descriptions of the Japanese diet from 39 selected articles, we were able to aggregate the descriptions into 16 categories from 33 factors. After performing a content analysis using a further aggregation of categories, we found that the top three applicable categories were soybeans/soybean-derived products, seafood, and vegetables; these were followed by rice and miso soup. Conclusion: The Japanese dietary content was found to be diverse based on an examination of epidemiological studies; however, we were able to aggregate the content into 16 categories. The Japanese diet is considered to be a dietary pattern that contains a combination of factors: the dietary staple, side dishes, and soup.
\end{abstract}

Key Words Japanese diet, systematic review, dietary pattern, character, content analysis

International interest in the Japanese diet has grown in recent years. Activities performed for the sake of the protection and succession of Japan's dietary culture have been implemented predominantly by the Ministry of Agriculture, Forestry and Fisheries (http://www.maff. go.jp/j/keikaku/syokubunka/culture/), and in 2013, the traditional dietary culture of the Japanese (washoku) was registered as a UNESCO Intangible Cultural Heritage (http://www.maff.go.jp/j/keikaku/syokubunka/ ich/). Furthermore, in 2015, Expo Milan was held, with the theme of Feeding the Planet, Energy for Life; the Japanese government was an official participant (http://www.maff.go.jp/j/shokusan/gaisyoku/milano/), and won the gold award (1). From a medical perspective, reports indicate that the Japanese diet reduces the total mortality rate (2) and is related to cancer prevention (3). In a report on dietary guidelines published in 2010 by the U.S. government, it was suggested that the Japanese diet reduces the risk of coronary artery dis-

E-mail: nzmsuzuki@gmail.com

* Present address: Department of Home Economics, Aichi Gakusen University, 28 Kamikawanari, Hegoshi-cho, Okazaki, Aichi 444-8520, Japan ease, and it demonstrated there is a strong interest in the Japanese diet (4).

An international joint study by seven countries around the globe reviewed country-specific food culture differences and found that southern European countries on the Mediterranean, such as Italy and Greece, had significantly lower mortality rates from ischemic heart disease compared with other European countries and the U.S., and the preventive effects of what is known as the Mediterranean diet attracted worldwide attention (5). Moreover, the DASH (Dietary Approaches to Stop Hypertension) diet $(6,7)$ is advocated for the prevention of hypertension in the U.S., with study reports currently ongoing (8).

In this way, these habits are a mainstay of the population's lifestyle, and the significance of evaluating the effect of dietary habits on health is increasing in terms of scientific and policy research. However, the aforementioned US dietary guidelines indicated that accumulation of research findings relating to the Japanese diet is delayed (4). The content of the Mediterranean diet has been organized and defined based on analytical methods (9) looking at dietary patterns comprehensively encompassing dietary lifestyle and evaluating 
Table 1. PECOS criteria used in the present systematic review.

\begin{tabular}{ll}
\hline \multicolumn{1}{c}{ Criteria } & \multicolumn{1}{c}{ Description } \\
\hline Population & Healthy human population with no restriction on age or sex. \\
& Studies in participants with disease, who were hospitalized or institutionalized were excluded. \\
Exposure & Diets defined as Japanese diet using dietary pattern analysis methods. \\
Comparisons & Diets defined as meals other than Japanese diet using dietary pattern analysis methods. \\
Outcomes & Outcome is not restricted. \\
Study designs & Cross-sectional study, cohort study, case-control study. \\
\hline
\end{tabular}

meal units, rather than simply looking at individual nutrients and foods (10). As a result, scientific investigations have been undertaken, including cohort research, interventional studies, and meta-analyses $(11,12)$, and these studies have clarified the effect on the reduction of risk factors for total mortality and cardiovascular diseases. Now, it is vital to move forward with ascertaining the characteristics and content of the Japanese diet to undertake research relating to its disease prevention and health promotion aspects.

In 2014, the National Agriculture and Food Research Organization, and the Ministry of Agriculture, Forestry and Fisheries inaugurated a research project relating to the evaluation of the Japanese diet in collaboration with medical and nutritional experts entitled Scientific and Multifaceted Verification of the Japanese Diet to Contribute to World Health, which forms part of the promotion of creating innovative technology, and thus, interdisciplinary verification of the Japanese diet is underway (http://nihonshoku.jp/). As part of this project, this research was implemented with the aim of evaluating and organizing Japanese dietary characteristics from an epidemiological perspective, mainly focusing on nutrition and diets.

\section{METHODS}

Literature was selected using the systematic review method (13), in which the main elements were extracted from the selected articles, compiled into categories, and the descriptions of the Japanese diet were analyzed. This report conforms to the reporting guidelines for qualitative research set out by O'Brien et al. (14).

Systematic Review.

Study eligibility criteria: Eligibility criteria are shown in Table 1. The eligibility and exclusion criteria were set before implementing the review. The target population was a healthy general population without disease, not hospitalized or institutionalized, and no limits were set for age or sex. The research design was an epidemiological study that was either cross-sectional, cohort, or case-control based. Moreover, in terms of the Japanese diet that was subject to evaluation, the types of target research were those that analyzed dietary patterns, after implementing food and dietary behavior surveys, and research that defined the dietary patterns of the Japanese diet. Non-English and non-Japanese articles, literature other than original articles (i.e., conference proceedings, letters, and opinions) and review articles were excluded from the study. No limitations were placed on publication date or format (e.g., pamphlet media or electronic media).

Data sources: We conducted our search using the following electronic databases: PubMed (since 1966), Web of Science (since 1945), Japan Medical Abstracts Society (Ichushi) (since 1977), JDream III (since 1981), and CiNii (since 2004). We investigated related books, journals, and government-issued reports. We also collected applicable literature from the reference lists for the aforementioned literature (final search date: October 25, 2015).

Search: We conducted our search using the search formula shown in Table 2. Other searches were conducted by changing the search formula shown in Table 2 to suit the format of each of the databases.

Literature selection: From the list of literature obtained in the search, two people independently sorted the titles and abstracts based on the criteria in Table 1 into "use", "exclude", and "undecided", and all the literature other than pieces excluded by both individuals was procured. We then further conferred on the candidate literature, and any literature exhibiting a difference of opinion was resolved by a third party.

Data extraction process: The authors created a data extraction sheet in advance, and one other individual checked the inputted data. The items in the data extraction sheet were set as literature characteristics (e.g., implementing country, location, study design, participant characteristics, key outcomes, and analysis methods of dietary patterns) and descriptions of the Japanese diet (e.g., explanations of foods included in Japanese dietary patterns). The analysis methods of the dietary patterns were organized as follows: deductive reasoning was used for methods that pre-defined the diets that the researcher was interested in during the research planning stage, while inductive reasoning was used for methods that defined the diets after the fact using methods such as factor analysis from the results of a food survey.

Analysis of data describing the Japanese diet.

Extraction and categorization of the main points in the Japanese diet: We partly reorganized the method used by Hsieh and Shannon (15), whose group extracted the main points related to the Japanese diet, and amalgamated them into categories based on similarities. More specifically, (i) we carefully reread the literature, (ii) identified sections where the names and details of Japa- 
Table 2. Example of the search strategy in PubMed for publications about the Japanese diet.

\begin{tabular}{|c|c|c|}
\hline \multicolumn{3}{|c|}{ PubMed search strategy, 25 October 2015 (no limits) } \\
\hline Search & Keywords & Results \\
\hline \#1 & ((“japan”[MeSH Terms] OR (“japan”[MeSH Terms] OR “japan”[All Fields]))) & 1013591 \\
\hline$\# 2$ & "japanese"[All Fields] & 496636 \\
\hline \#3 & $(\# 1$ OR \#2) & 1359149 \\
\hline$\# 4$ & "food"[MeSH Terms] & 561095 \\
\hline \#5 & “diet”[MeSH Terms] & 205955 \\
\hline \#6 & "meals"[MeSH Terms] & 1571 \\
\hline \#7 & "food quality"[MeSH Terms] & 71211 \\
\hline \#8 & "eating”[MeSH Terms] & 60138 \\
\hline$\# 9$ & "nutrition policy"[MeSH Terms] & 7145 \\
\hline \#10 & "nutritional requirements"[MeSH Terms] & 17733 \\
\hline \#11 & (\#4 OR \#5 OR \#6 OR \#7 OR \#8 OR \#9 OR \#10) & 773611 \\
\hline \#12 & "food habits"[MeSH Terms] & 24030 \\
\hline \#13 & "feeding behavior" [MeSh Terms] & 129499 \\
\hline$\# 14$ & (\#12 OR \#13) & 129499 \\
\hline \#15 & $(\# 11$ OR \#14) & 831623 \\
\hline \#16 & $\begin{array}{l}\text { ((index*[tiab] OR score*[tiab] OR indexes[tiab] OR indices[tiab] OR scoring[tiab]) AND (dietary } \\
\text { score*[All Fields] OR adequacy index*[All Fields] OR Diet Quality Index*[All Fields] OR Food Score*[All } \\
\text { Fields] OR Diet Score*[All Fields] OR Dietary Pattern Score*[All Fields] OR "healthy eating index"[All } \\
\text { Fields]) OR ("Diet Quality Index"[All Fields] OR "Recommended Food Score"[All Fields] OR "Eating } \\
\text { Plan Score"[All Fields] OR "Diet Score"[All Fields] OR "Dietary Pattern Score"[All Fields])) }\end{array}$ & 1470 \\
\hline$\# 17$ & $\begin{array}{l}\text { ((“diet quality”[All Fields] OR dietary[tiab] OR nutrient*[All Fields] OR eating[tiab] OR food[tiab] } \\
\text { OR diet[tiab] OR diet[mh]) AND (pattern*[All Fields] OR habit*[All Fields] OR profile*[All Fields] OR } \\
\text { recommendation*[All Fields] OR guideline*[All Fields]) AND ("Principal component analysis"[tiab] } \\
\text { OR "Factor analysis”[tiab] OR "Cluster analysis”[tiab] OR "rank regression"[tiab] OR "Discriminant } \\
\text { analysis"[tiab] OR "Cluster Analysis”[Mesh Terms] OR "Factor Analysis, Statistical”[Mesh Terms] OR } \\
\text { "Principal Component Analysis”[Mesh Terms] OR "Discriminant Analysis"[Mesh Terms] OR "Regres- } \\
\text { sion Analysis"[Mesh Terms])) }\end{array}$ & 8194 \\
\hline \#18 & $\begin{array}{l}\text { (("diet quality”[All Fields] OR dietary pattern*[All Fields] OR diet pattern*[All Fields] OR eating } \\
\text { pattern*[All Fields] OR food pattern*[All Fields] OR eating habit*[All Fields] OR dietary habit*[All } \\
\text { Fields] OR food habit*[All Fields] OR dietary profile*[All Fields] OR food profile*[All Fields] OR } \\
\text { diet profile*[All Fields] OR eating profile*[All Fields] OR dietary guideline*[All Fields] OR dietary } \\
\text { recommendation*[All Fields] OR food intake pattern*[All Fields] OR dietary intake pattern*[All Fields] } \\
\text { OR diet pattern*[All Fields])) }\end{array}$ & 38995 \\
\hline \#19 & $\begin{array}{l}\text { ((“Guideline Adherence”[Mesh Terms] AND (“diet”[All Fields] OR "food”[All Fields] OR "eating”[All } \\
\text { Fields] OR "eat”[All Fields] OR "dietary"[All Fields] OR "feeding”[All Fields] OR "nutrition”[All Fields] } \\
\text { OR "nutrient*”[All Fields]) OR "adherence"[All Fields]) AND (nutrient* OR nutrition OR diet OR } \\
\text { dietary OR food OR eat OR eating) AND (guideline* OR guidance OR recommendation*)) }\end{array}$ & 2749 \\
\hline \#20 & (\#16 OR \#17 OR \#18 OR \#19) & 45782 \\
\hline \#21 & $\begin{array}{l}\text { ((“japanese diet”[All Fields] OR “japanese diets"[All Fields] OR "japanese style diet”[All Fields] OR } \\
\text { "japanese style diets”[All Fields] OR "japanese food”[All Fields] OR "japanese foods”[All Fields] OR } \\
\text { "japanese style food”[All Fields] OR "japanese style foods"[All Fields])) }\end{array}$ & 360 \\
\hline \#22 & $\begin{array}{l}(((\text { "japan"[All Fields] OR "japanese"[All Fields]) AND ("traditional diet”[All Fields] OR "traditional } \\
\text { diets"[All Fields] OR "traditional food"[All Fields] OR "traditional foods"[All Fields]))) }\end{array}$ & 85 \\
\hline \#23 & $(\# 21$ OR \#22) & 441 \\
\hline \#24 & (\#3 AND \#15) & 42053 \\
\hline \#25 & (\#24 OR \#23) & 42205 \\
\hline$\# 26$ & (\#25 AND \#20) & 1572 \\
\hline
\end{tabular}

nese diet were described, (iii) focused on descriptions relating to explanation of dietary patterns (i.e., food, food groups, and cooking) and extracted those descriptions as factors, (iv) summarized the extracted factors as a list for each of the literature pieces, (v) grouped similar factors based on the list of factors and created categories, (vi) checked that there were no inconsistencies between the different categories created, and finally, (vii) we aggregated the data into categories. We reorganized parts of the procedures for (ii), (iii), and (iv) reported by Hsieh and Shannon (15) for this investigation. Furthermore, factor extraction and category aggregation were investigated based on the opinion of our supervisor (a physician specializing in epidemiology and public health, and experienced in qualitative research).

Analyzing the content of descriptions of the Japanese diet: We performed the content analysis (16) using the aggregated categories. Specifically, we reread the descrip- 


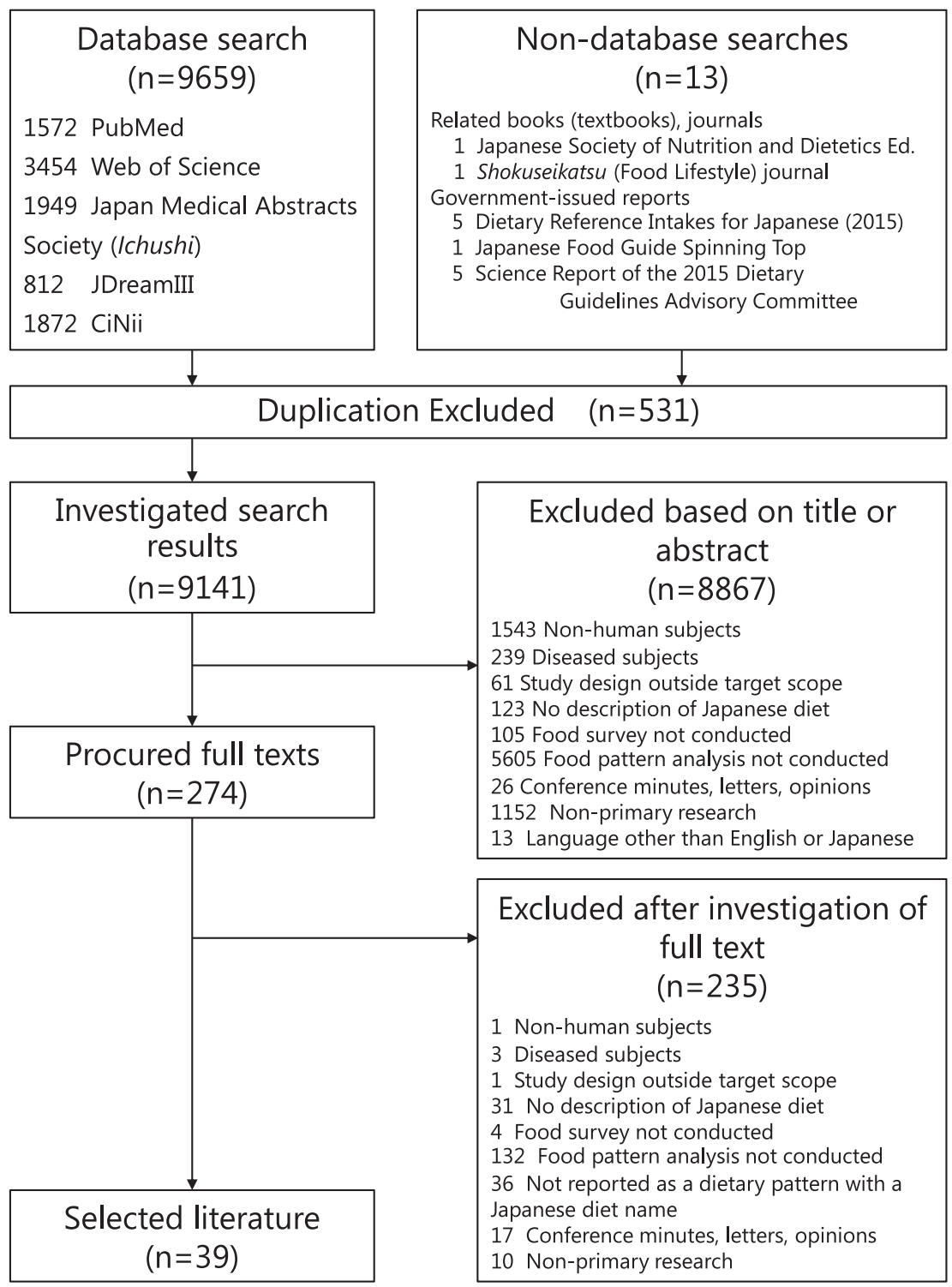

Fig. 1. Flow chart summary of the search strategy.

tive data on the Japanese diet obtained from each article and confirmed that the description data were applicable to the aggregated category. Following this, one more individual (a registered dietitian experienced in qualitative research) checked the inputted data. After the data were checked, agreement was reached on any difference of opinion through discussion between the two parties.

\section{RESULTS}

Literature search and selection of target literature

We conducted a database search and a non-database search, and once we excluded any duplication of complete literature matches, there were 9,141 articles. We then sorted the literature using eligibility criteria based on titles and abstracts. As a result, we procured the full text for 274 articles. We then checked the full text of these articles and eventually selected 39 articles. Figure 1 shows the flowchart for literature selection.

Literature characteristics

The list of the 39 selected articles is shown in Table
3. The regions where the studies were implemented encompassed 5 countries, and the implementation locations were as follows: 23 articles were region based, 10 articles were occupation based, 5 articles were school based, and 1 was hospital based. In terms of research design, 25 articles were cross-sectional studies, 11 articles were cohort studies, and 3 articles were case-control studies. There were articles in which the age of the subjects ranged from $18.1 \mathrm{y}$ (mean) to $69.8 \mathrm{y}$ (mean), and there were 6 articles in which the age of the subjects was not stated. Factor analysis was adopted in 35 articles as the analysis method for dietary patterns, while 3 articles used score analysis, and 1 article used reduced rank regression analysis.

Description of the Japanese diet

The descriptions of the Japanese diet from 39 articles are shown as partial excerpts in Table 4.

Japanese diet factors and categories

We obtained 40 descriptions of the Japanese diet from 39 articles. One article (literature reference 36, Kaku- 
Table 3. Study characteristics of eligible studies included in this review.

\begin{tabular}{|c|c|c|c|c|c|c|c|}
\hline Reference & Country & Setting & Study design & $\begin{array}{c}n=\text { subjects } \\
(\% \text { female })\end{array}$ & Age* $^{*}$ & Outcome & $\begin{array}{l}\text { Analytic methods } \\
\text { of dietary pattern }\end{array}$ \\
\hline 1 Hankin 1975 & $\begin{array}{l}\text { United } \\
\text { States } \\
\text { (Hawaii) }\end{array}$ & Region & Cross-sectional & $6,663(0)$ & ND & Dietary intake & Index Analysis \\
\hline 2 Nomura 1978 & $\begin{array}{l}\text { United } \\
\text { States } \\
\text { (Hawaii) }\end{array}$ & Region & Case-control & $6,860(0)$ & ND & Breast cancer in spouse & Factor Analysis \\
\hline 3 Okimasu 1981 & $\begin{array}{l}\text { Japan, } \\
\text { Brazil }\end{array}$ & Region & Cross-sectional & $289(100)$ & ND & Dietary intake & Factor Analysis \\
\hline $4 \begin{array}{l}\text { Kumasawa } \\
1983\end{array}$ & Japan & $\begin{array}{l}\text { Elementary } \\
\text { school }\end{array}$ & Cross-sectional & $619(50)$ & ND & Dietary intake & Factor Analysis \\
\hline $\begin{array}{l}5 \begin{array}{l}\text { Nakamura } \\
2001\end{array} \\
\end{array}$ & Japan & Region & Case-control & $20,477(45)$ & $20-79$ & Sudden deafness & Factor Analysis \\
\hline 6 Mizoue 2005 & Japan & Occupational & Cross-sectional & $1,341(0)$ & ND & Colon cancer & Factor Analysis \\
\hline 7 Mizoue 2006 & Japan & Occupational & Cross-sectional & $2,106(0)$ & $46.4(3.7)$ & Type II diabetes & Factor Analysis \\
\hline 8 Okubo 2006 & Japan & Region & Cross-sectional & $291(100)$ & $46.4(3.7)$ & Bone density & Factor Analysis \\
\hline 9 Hirose 2007 & Japan & Hospital & Case-control & $24,218(100)$ & $40-79$ & Breast cancer & Factor Analysis \\
\hline 10 Okubo 2007 & Japan & School & Cross-sectional & $3,770(100)$ & $18.1(0.3)$ & Functional constipation & Factor Analysis \\
\hline 11 Pierce 2007 & $\begin{array}{l}\text { United } \\
\text { States }\end{array}$ & Region & Cross-sectional & $496(56)$ & $\begin{array}{l}\text { Nisei } 69.8(8.0) \text {, } \\
\text { Sansei } 41.8(8.0)\end{array}$ & BMI, CRP & Factor Analysis \\
\hline 12 Shimazu 2007 & Japan & Region & Cohort & $40,547(\mathrm{ND})$ & $40-79$ & $\begin{array}{l}\text { Mortality rate of cardio- } \\
\text { vascular disease patients }\end{array}$ & Factor Analysis \\
\hline 13 Okubo 2008 & Japan & School & Cross-sectional & $3,760(100)$ & $18-20$ & BMI & Factor Analysis \\
\hline $\begin{array}{l}14 \begin{array}{l}\text { Nakamura } \\
2009\end{array} \\
\end{array}$ & Japan & Region & Cohort & $9,086(56)$ & $30-\mathrm{ND}$ & Mortality rate & Index Analysis \\
\hline 15 Gimeno 2010 & Brazil & Region & Cross-sectional & $1,283(54)$ & $57.1(12.4)$ & $\begin{array}{l}\text { Anthropometric measure- } \\
\text { ments, Lifestyle-related } \\
\text { diseases }\end{array}$ & Factor Analysis \\
\hline 16 Monma 2010 & Japan & Region & Cohort & $877(56)$ & 70-ND & Bone fracture from falls & Factor Analysis \\
\hline 17 Nanri 2010 & Japan & Occupational & Cross-sectional & $521(41)$ & $21-67$ & Depression & Factor Analysis \\
\hline 18 Okubo 2010 & Japan & Region & Cross-sectional & $184(50)$ & $31-76$ & Dietary intake & Factor Analysis \\
\hline 19 Zenitani 2010 & Japan & Occupational & Cross-sectional & $634(26)$ & $39.5(8.3)$ & BMI & Factor Analysis \\
\hline 20 Miyake 2011 & Japan & Region & Cohort & $763(100)$ & $30.0(4.0)$ & Asthma and eczema & Factor Analysis \\
\hline 21 Okubo 2011 & Japan & Region & Cohort & $865(100)$ & $29.9(4.0)$ & Post-natal depression & Factor Analysis \\
\hline 22 Kumeda 2011 & Japan & Region & Cross-sectional & $763(62)$ & $56.3(13.1)$ & Irritable bowel syndrome & Factor Analysis \\
\hline 23 Akter 2012 & Japan & Occupational & Cross-sectional & $456(40)$ & $21-67$ & CRP & Factor Analysis \\
\hline 24 Guo 2012 & Japan & Occupational & Cross-sectional & $702(0.0)$ & $\begin{array}{l}44.5^{\dagger} \\
{[37.8,54.2]}\end{array}$ & Adiponectin levels & Factor Analysis \\
\hline 25 Nanri 2012 & Japan & Region & Cross-sectional & $498(51)$ & $40-69$ & Dietary intake & Factor Analysis \\
\hline 26 Yap 2012 & $\begin{array}{l}\text { Japan, } \\
\text { Malaysia }\end{array}$ & Region & Cross-sectional & $136(39)$ & $43.0(11.0)$ & Gene polymorphism & Factor Analysis \\
\hline 27 Akter 2013 & Japan & Occupational & Cross-sectional & $460(38)$ & $21-67$ & Lifestyle-related diseases & Factor Analysis \\
\hline $28 \begin{array}{l}\text { Morinaka } \\
2013\end{array}$ & $\begin{array}{l}\text { Japan, } \\
\text { Poland }\end{array}$ & University & Cross-sectional & $100(100)$ & $19.6(1.4)$ & Dietary intake & Factor Analysis \\
\hline 29 Nanri 2013 & Japan & Region & Cohort & $89,037(54)$ & $40-69$ & Suicide & Factor Analysis \\
\hline 30 Nanri 2013 & Japan & Region & Cohort & $64,705(57)$ & $40-69$ & Type II diabetes & Factor Analysis \\
\hline 31 Ozawa 2013 & Japan & Region & Cohort & $1,006(57)$ & $68.0(\mathrm{ND})$ & Dementia & $\begin{array}{l}\text { Reduced Rank } \\
\text { Regression }\end{array}$ \\
\hline 32 Suzuki 2013 & Japan & Occupational & Cross-sectional & $2,266(11)$ & $21-65$ & Depression & Factor Analysis \\
\hline 33 Guo 2014 & Japan & Region & Cross-sectional & $1,096(23)$ & $46.2(11.2)$ & Lifestyle-related diseases & Factor Analysis \\
\hline 34 Kumagai 2014 & Japan & Region & Cohort & $44,097(52)$ & $40-79$ & Colon cancer & Factor Analysis \\
\hline 35 Tomata 2014 & Japan & Region & Cohort & $14,260(55)$ & $73.9(6.0)$ & Life dysfunction & Factor Analysis \\
\hline 36 Kakutani 2015 & Japan & University & Cross-sectional & $664(72)$ & $18-24$ & Dietary intake & Index Analysis \\
\hline 37 Kashino 2015 & Japan & Occupational & Cross-sectional & $509(42)$ & $20-65$ & Adipokine level & Factor Analysis \\
\hline 38 Kurotani 2015 & Japan & Occupational & Cross-sectional & $2,025(11)$ & $18-70$ & Sleeping disorder & Factor Analysis \\
\hline 39 Niu 2015 & Japan & Region & Cohort & $(25)$ & ND & $\begin{array}{l}\text { BMI, blood pressure, } \\
\text { fasting blood sugar, blood } \\
\text { lipids }\end{array}$ & Factor Analysis \\
\hline
\end{tabular}


Table 4. Definition of Japanese diet (Excerpt).

Reference

Definition

Then, we defined seven components from the nutritional survey to measure a healthy reduced-salt Japanese diet. The components included egg intake $\leqq 2$ eggs/wk, fish intake once or more often in $2 \mathrm{~d}$, meat intake $\leqq 2$ times/wk, tsukemono intake once or more often per day, infrequent intake of soup with noodles, use of low-salt soya sauce and occasional drinking.

14 Nakamura 2009

The third pattern was characterized by: high intakes of fish and shellfish, natto (fermented soy-

38 Kurotani 2015 beans), buckwheat noodles and pickled green leafy vegetables, and low intakes of red meat; this pattern was named the traditional Japanese dietary pattern.

Table 5. Code and Category of Japanese diet in this review.

\begin{tabular}{|c|c|}
\hline Codes & Categories \\
\hline Rice & Rice \\
\hline $\begin{array}{l}\text { Udon } \\
\text { Soba }\end{array}$ & Soba, udon \\
\hline $\begin{array}{l}\text { Seafood } \\
\text { Fish } \\
\text { Salmon } \\
\text { Kamaboko (fish paste) } \\
\text { Mackerel } \\
\text { Shellfish }\end{array}$ & Seafood \\
\hline Meat & Meat \\
\hline Eggs & Eggs \\
\hline $\begin{array}{l}\text { Soy bean } \\
\text { Soy bean-derived products } \\
\text { Tofu } \\
\text { Natto }\end{array}$ & $\begin{array}{l}\text { Soy beans/Soy bean-derived } \\
\text { products }\end{array}$ \\
\hline $\begin{array}{l}\text { Vegetables } \\
\text { Green and yellow vegetables } \\
\text { Light-colored vegetables } \\
\text { Carrot } \\
\text { Pumpkin } \\
\text { Cabbage }\end{array}$ & Vegetables \\
\hline Seaweed & Seaweed \\
\hline Mushrooms & Mushroom \\
\hline Potato & Potatoes \\
\hline Pickles & Pickles \\
\hline Miso soup & Miso soup \\
\hline $\begin{array}{l}\text { Milk } \\
\text { Dairy products }\end{array}$ & Milk/dairy products \\
\hline Green tea & Green tea \\
\hline Fruit & Fruit \\
\hline $\begin{array}{l}\text { Miso } \\
\text { Soy sauce } \\
\text { Reduced-salt soy sauce }\end{array}$ & Miso, soy sauce \\
\hline
\end{tabular}

tani 2015) described the Japanese diet as a "combination of dietary staples, main dishes, and side dishes", but this was excluded from analysis, as it did not describe the names of the food or the manner of cooking. As a result, 33 factors were extracted from the 39 pieces of descriptive data. Next, the 33 extracted factors were aggregated into 16 categories referencing food groups in standard tables of food composition in Japan (Table 5) (17).

Analyzing the content of data describing the Japanese diet

The results of content analysis of the 39 descriptions of the Japanese diet based on 16 categories are shown in Table 6.

The number of applicable categories for the 39 descriptions ranged from two categories (literature reference 9: Hirose 2007; literature reference 18: Okubo 2010; literature reference 19: Zenitani 2010; reference 29: Nanri 2013) to 8 categories (literature reference 2: Nomura 1978; literature reference 35: Tomata 2014), and both the median and mode category values were 5 .

The number of categories applicable to the descriptions of the Japanese diet is shown in Fig. 2. The top three categories with the highest number of descriptions were soybeans/soybean-derived products (27 descriptions), seafood (23 descriptions), and vegetables (20 descriptions). This was followed by rice (18 descriptions) and miso soup (16 descriptions).

\section{DISCUSSION}

As a result of analyzing the descriptions of the Japanese diet from 39 articles adopted through systematic review, we were able to aggregate the descriptions into 16 categories from 33 factors. After performing the content analysis using further aggregated categories, we found that the top 3 applicable number of categories were soybeans/soybean-derived products, seafood, and vegetables, followed by rice and miso soup.

Factor analysis was used in 35 of the 39 articles obtained, and 1 article used reduced rank regression analysis. There were broadly two methods used for defining dietary patterns $(18,19)$. Deductive reasoning methods were used where the researcher pre-defined the Japanese diet at the research planning stage, and inductive reasoning methods was used where the Japanese diet was defined after the fact using methods such as factor analysis from the results of a food survey. The former is used in cohort studies for hypothesis testing, while 
Table 6. Categories found in definition of Japanese diet.

\section{Aggregated 16 categories}

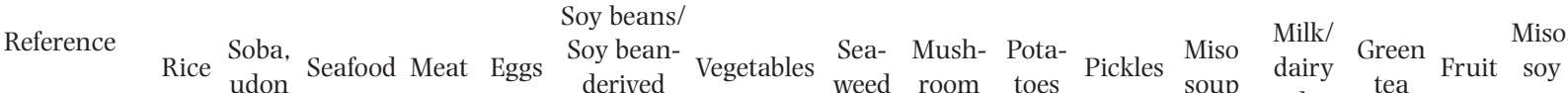

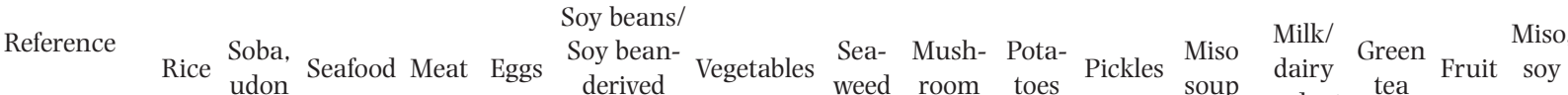

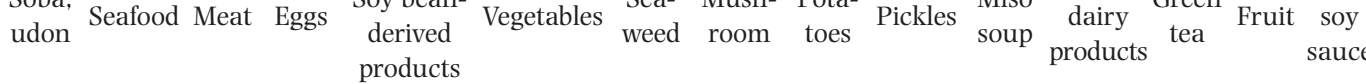

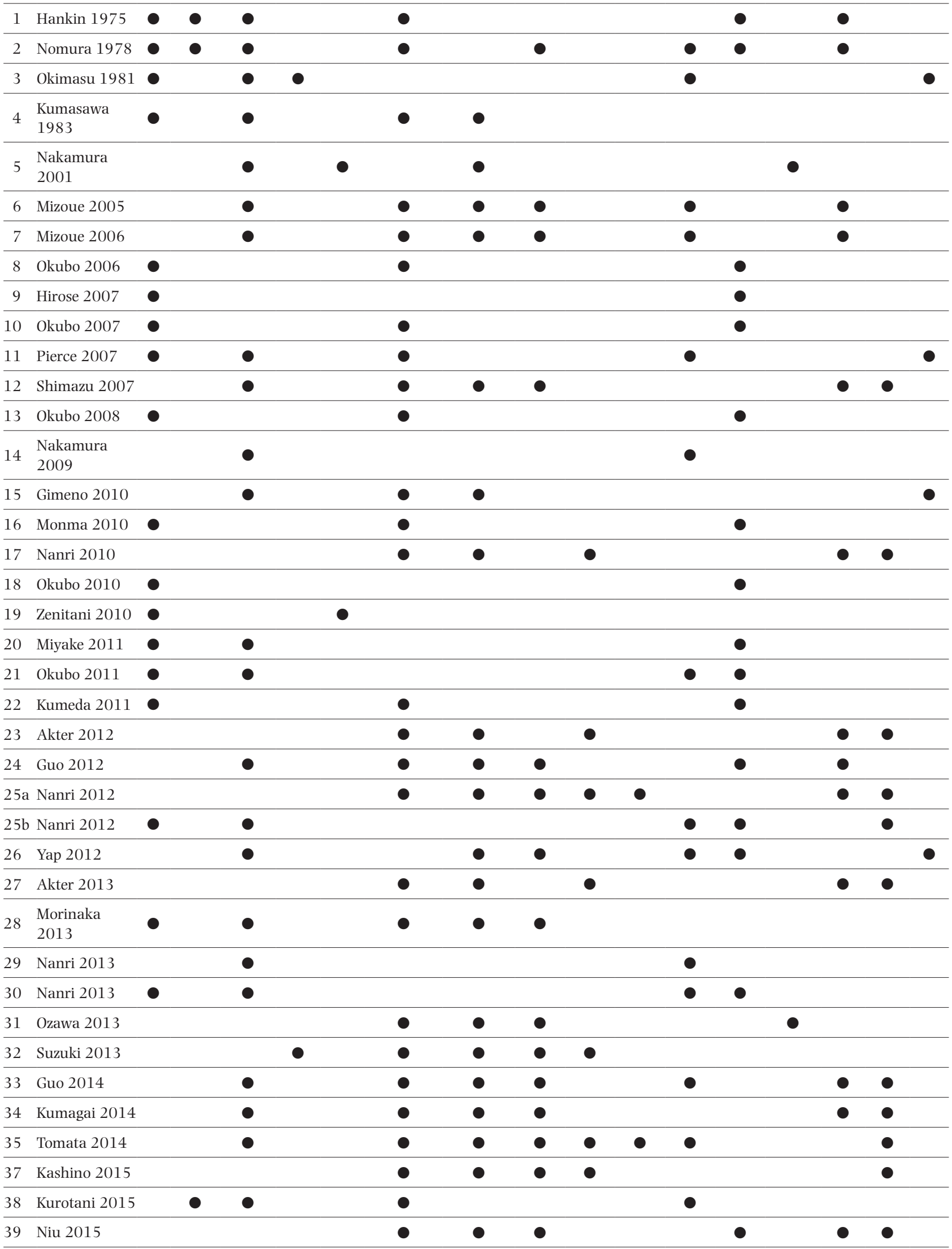

-Description found. 


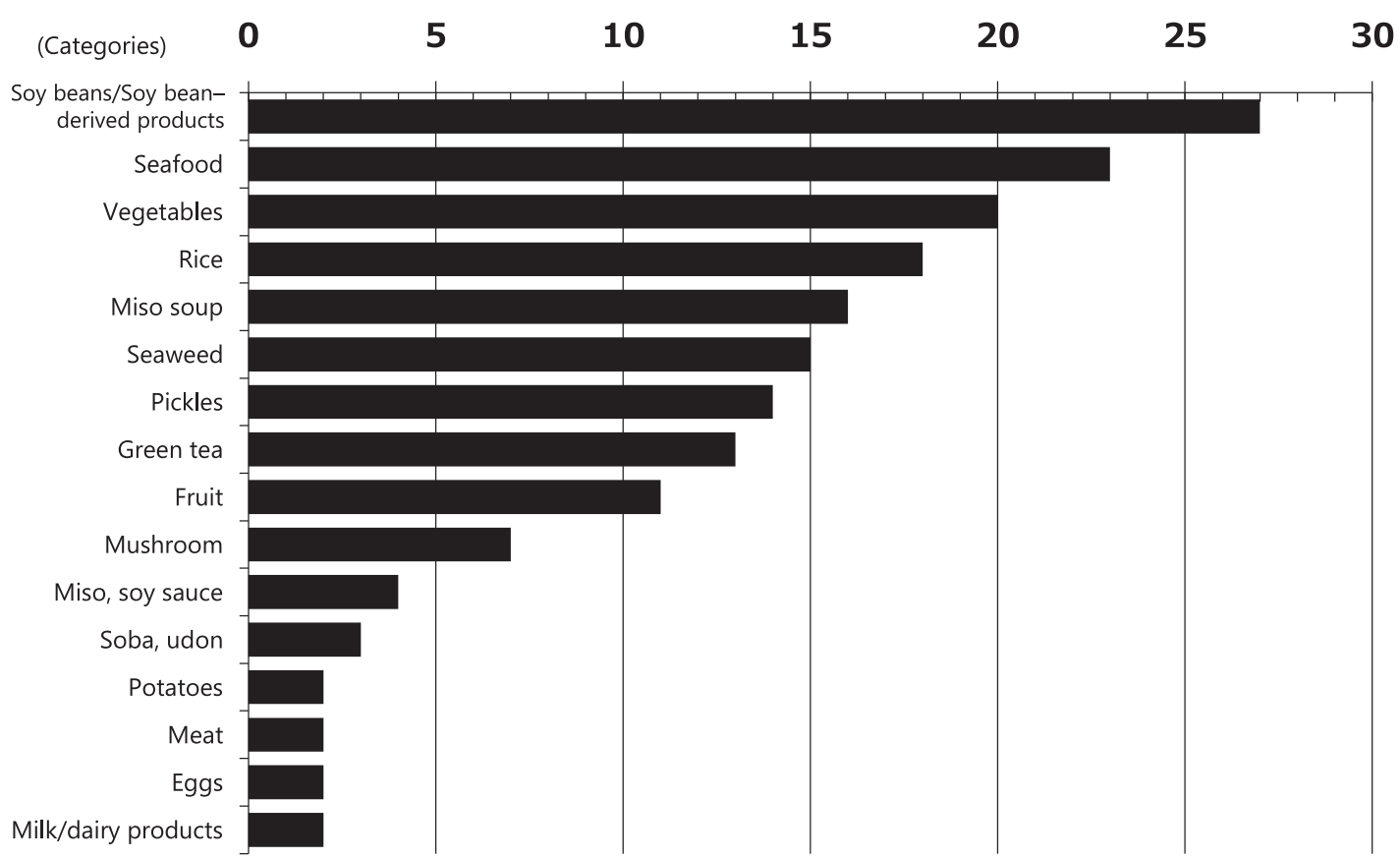

Fig. 2. The number of correspondences with the categories from content analysis.

the latter is typified by reports that conducted factor analysis on cross-sectional study data for food surveys. Problems indicated with inductive reasoning include the researcher's subjective views becoming involved in the naming of the dietary patterns for the obtained results (20). Specifically, among different researchers, the same diet content may be defined using different dietary pattern names, or conversely, different diet content may be defined using the same name. In other words, even in the results obtained in this study, Japanese diets given the same name does not necessarily guarantee that the diets have the same content. Therefore, it is difficult to acquire common opinions on the Japanese diet from the accumulated results of factor analysis where there tends to be arbitrary interpretation of the results by researchers.

If we consider each description of the Japanese diet from the perspective of dietary patterns, which are a combination of food and cooking, then the descriptions include a combination of multiple factors (dietary staples, soups, and side dishes). There are multiple explanations of the Japanese diet, including perspectives on food culture that include viewpoints on historical changes, ethnic, ideological and economic factors (http://www. maff.go.jp/j/keikaku/syokubunka/culture/wasyoku. $\mathrm{html}$ ). One of the explanations about the Japanese diet is the basic pattern of "one soup and three side dishes," advocated by the Ministry of Agriculture, Forestry and Fisheries, which consists of rice and soup and a side dish (main dish and side dish). This description is the Japanese dietary pattern considered from the perspective of the composition of one meal (21). The descriptions of the Japanese diet in this study fit multiple categories. From the perspective of the meal composition for the Japanese dietary pattern for these categories, there were 10 articles where the data were applicable to multiple categories as a combination of the staple food, one or more side dishes and soup (literature reference 1: Hankin 1975; literature reference 2: Nomura 1978; literature reference 8: Okubo 2006; literature reference 10: Okubo 2007; literature reference 13: Okubo 2008; literature reference 16: Monma 2010; literature reference 20: Miyake 2011; literature reference 21: Okubo 2011 literature reference 22: Kumeta 2011; literature reference 25: Nanri 2012). These articles comprehensively reported the diet content as the dietary pattern known as the Japanese diet, and after confirming multiple categories of each description of the Japanese diet, we considered that it is a dietary pattern comprising multiple factors including the dietary staple, side dishes and soup. The basic pattern of "one soup and three side dishes", advocated by the Ministry of Agriculture, Forestry and Fisheries, which is based on a typical traditional Japanese diet, is also supported by the findings of an epidemiological study on nutrition and diet. In this way, it could be presented as a common understanding of the Japanese diet.

We hope that these findings can be used as basic data for research in other research projects (http://nihonshoku.jp/) (epidemiological studies to create a Japanese dietary score, etc.), or they can be used as material for discussion with specialists in other fields including cooking, food culture and history for multifaceted investigation of the Japanese diet.

\section{Limitations}

This study targeted epidemiological studies on the topic of nutrition and diet with the aim of organizing the characteristics of the Japanese diet to verify its relationship with health, including health promotion and disease prevention in target populations. Therefore, we have not necessarily comprehensively incorporated all the targets involved in the Japanese diet. It is also essen- 
tial to be careful when interpreting the categories with a large number of reports as having a high degree of importance. These categories should only be viewed as having the range that contains the common items recognized as Japanese dietary characteristics.

\section{CONCLUSION}

Japanese diet content was diverse when using epidemiological studies; however, we aggregated the content into 16 categories. We found that the top 3 categories were soybeans/soybean-derived products, seafood, and vegetables, followed by rice and miso soup. The Japanese diet is considered to be a dietary pattern that contains a combination of factors: the dietary staple, side dishes, and soup.

\section{Funding sources}

Funding was provided by the integrated research for agriculture and interdisciplinary fields (fiscal year 2014-2016) "Evaluation of the Japanese diet through collaboration of medical and nutritional experts (representative researcher: Nobuya Inagaki).”

\section{Acknowledgments}

We would like to express our heartfelt gratitude to all of the researchers at the Kyoto University School of Public Health.

\section{REFERENCES}

1) Yamano Y. 2015. Japanese pavillion wins gold medal in EXPO 2015 MILANO. Asahi Shimbun. Oct 31 p. 2 (in Japanese).

2) Nakamura Y, Ueshima H, Okamura T, Kadowaki T, Hayakawa T, Kita Y, Abbott RD, Okayama A. 2009. A Japanese diet and 19-year mortality: national integrated project for prospective observation of non-communicable diseases and its trends in the aged, 1980. Br J Nutr 101: 1696-1705.

3) Tsugane S, Sawada N. 2014. The JPHC study: design and some findings on the typical Japanese diet. Jpn J Clin Oncol 44: 777-782.

4) Dietary Guidelines Advisory Committee. 2010. Report of the Dietary Guidelines Advisory Committee on the Dietary Guidelines for Americans, 2010, to the Secretary of Agriculture and the Secretary of Health and Human Services. U.S.

5) Menotti A, Puddu PE. 2015. How the Seven Countries Study contributed to the definition and development of the Mediterranean diet concept: a 50-year journey. Nutr Metab Cardiovasc Dis 25: 245-252.

6) US Department of Health and Human Services, National Institutes of Health, National Heart, Lung and BI. Facts about the DASH eating plan. (http://www.nhlbi.nih. gov/files/docs/public/heart/new_dash.pdf. Accessed June 15, 2017.)

7) Sacks FM, Svetkey LP, Vollmer WM, Appel LJ, Bray GA, Harsha D, Obarzanek E, Conlin PR, Miller ER 3rd, Simons-Morton DG, Karanja N, Lin PH; DASH-Sodium Collaborative Research Group. 2001. Effects on blood pressure of reduced dietary sodium and the Dietary Approaches to Stop Hypertension (DASH) Diet. N Engl J Med 344: 3-10.

8) Saneei P, Salehi-Abargouei A, Esmaillzadeh A, Azadbakht L. 2014. Influence of Dietary Approaches to Stop Hypertension (DASH) diet on blood pressure: A systematic review and meta-analysis on randomized controlled trials. Nutr Metab Cardiovasc Dis 24: 1253-1261.

9) Hu FB. 2002. Dietary pattern analysis: a new direction in nutritional epidemiology. Curr Opin Lipidol 13: 3-9.

10) Serra-Majem L, Trichopoulou A, Ngo de la Cruz J, Cervera P, García Alvarez A, La Vecchia C, Lemtouni A, Trichopoulos D. 2004. Does the definition of the Mediterranean diet need to be updated? Public Health Nutr 7: 927-929.

11) Sofi F, Macchi C, Abbate R, Gensini GF, Casini A. 2014. Mediterranean diet and health status: an updated metaanalysis and a proposal for a literature-based adherence score. Public Health Nutr 17: 2769-2782.

12) Rees K, Hartley L, Flowers N, Clarke A, Hooper L, Thorogood M, Stranges S. 2013. "Mediterranean" dietary pattern for the primary prevention of cardiovascular disease. Cochrane Database Syst Rev 8: CD009825.

13) Stroup DF, Berlin JA, Morton SC, Olkin I, Williamson GD, Rennie D, Moher D, Becker BJ, Sipe TA, Thacker SB. 2000. Meta-analysis of observational studies in epidemiology: a proposal for reporting. Meta-analysis of Observational Studies in Epidemiology (MOOSE) group. JAMA 283: 2008-2012.

14) O'Brien BC, Harris IB, Beckman TJ, Reed DA, Cook DA. 2014. Standards for reporting qualitative research: a synthesis of recommendations. Acad Med 89: 1245-1251.

15) Hsieh H-F, Shannon SE. 2005. Three approaches to qualitative content analysis. Qual Health Res 15: $1277-1288$.

16) Rice PL, Kihara M, Kihara M. 2012. Current Medical Research Methods: Qualitative and Quantitative Methods, Mixed Methods, EBP. Medical Science International, Tokyo (in Japanese).

17) Ministry of Education, Culture, Sports, Science and Technology. Standard tables of food composition in Japan 2015 Edition (7th Revision). 2015 (in Japanese). (http://www.mext.go.jp/a_menu/syokuhinseibun/ 1365297.htm. Accessed June 15, 2017.)

18) Hu FB, Kobayashi M, Yatsuya H, Kobayashi K. 2010. Epidemiology of Obesity (Hu FB, ed). University of Nagoya Press, Nagoya.

19) Nutrition Evidence Library. A series of systematic reviews on the relationship between dietary patterns and health outcomes. 2014. (https://www.cnpp.usda. gov/sites/default/files/usda_nutrition_evidence_flbrary/ DietaryPatternsExecutiveSummary.pdf. Accessed June 15, 2017.)

20) Willett W. 2013. Nutritional Epidemiology, 3rd ed. Oxford University Press, New York.

21) Ministry of Agriculture, Forestry and Fisheries/Material distributed by the advisory council for measures to promote dietary education 15 July 2014_"Japanese dietary lifestyle" (in Japanese). (http://www.maff.go.jp/j/ syokuiku/pdf/2_s_2.pdf. Accessed June 15, 2017.) 